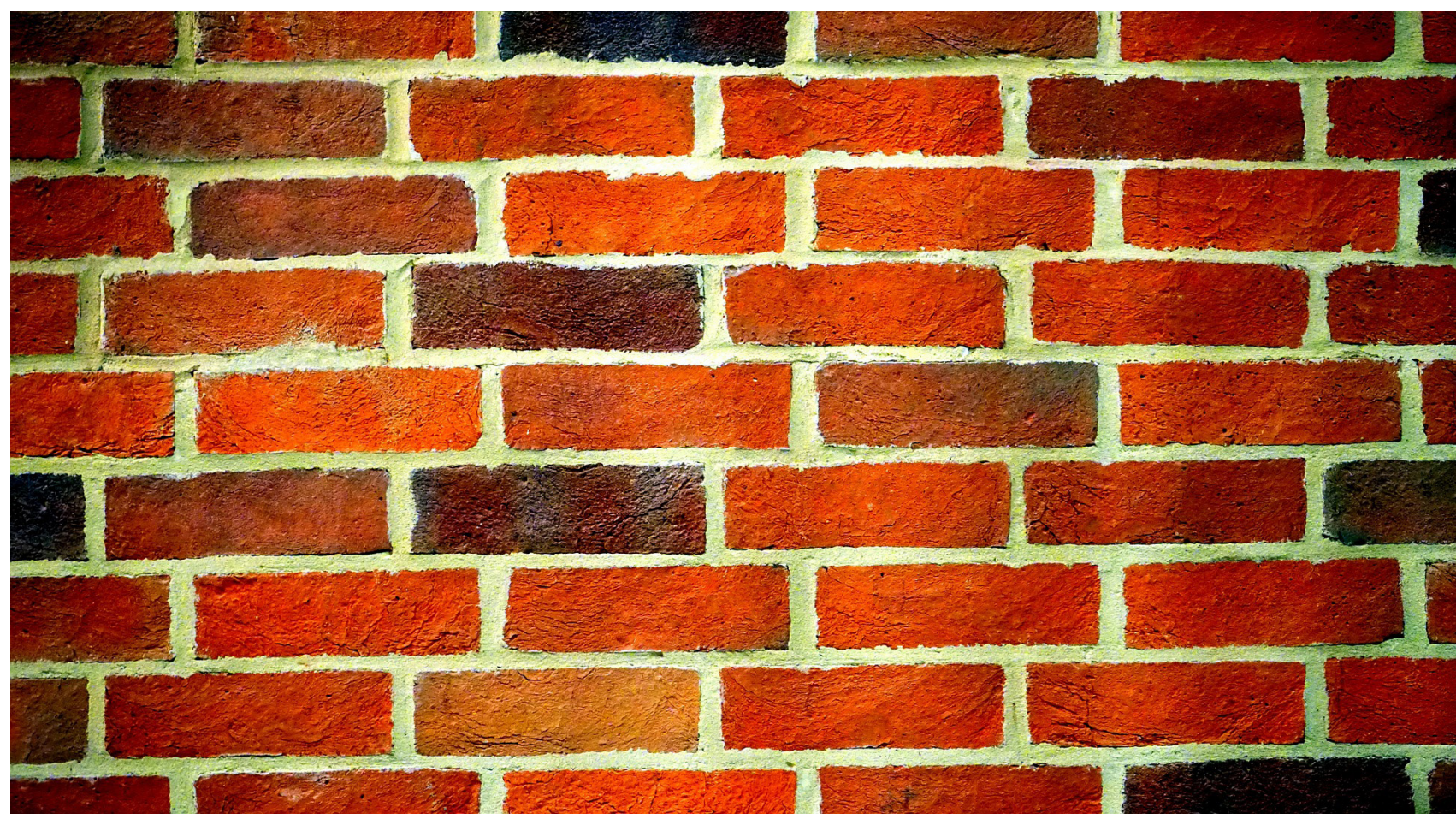

\title{
Evaluación de mezclas de arcilla adicionando componentes tecnológicos para la fabricación de bloques de construcción
}

\section{Evaluation of clay mixtures adding technological components for the manufacture of building blocks}

Andrés Felipe Ruíz

Tecnólogo en Obras Civiles, andresfeliperj@ufps.edu.co, Universidad Francisco de Paula Santander, Cúcuta, Colombia

Carlos Javier Peñaranda

Tecnólogo en Obras Civiles, carlosjaviermcp@gmail.com, Universidad Francisco de Paula Santander, Cúcuta, Colombia 


\section{Resumen}

La construcción ha estado en busca de la optimización de sus procesos y en el mejoramiento de los materiales y sus aplicaciones a través de la reutilización de productos como complementos para el mejoramiento de los materiales de construcción con el fin de generar una construcción sostenible que sea amigable con el medio ambiente, como lo plantea la economía circular la cual es un concepto relacionado con el aprovechamiento de los recursos. Una economía lineal plantea el aprovechamiento de los recursos desde un principio hasta un final, mientras que la circular trata de reutilizarlos insertándolos de nuevo en el proceso, esto podría entenderse también como la optimización del ciclo de vida. El presente artículo describe la evaluación de componentes tecnológicos utilizados en la mezcla de la elaboración de bloques de arcilla de construcción, tales como hojas de árboles y ceniza de raquis de palma africana, teniendo como objetivo estimar su factibilidad en costos y realizar un diagnóstico de las diferentes propiedades que proporcionan estos componentes a la mezcla, así como los requisitos que deben cumplir los bloques de arcilla según la norma sismorresistente colombiana NSR10.

Palabras clave: Evaluación, reutilización, bloques de arcilla, factibilidad, medio ambiente, diagnostico.

\section{Abstract}

Construction has been in search of the optimization of its processes and in the improvement of materials and their applications through the reuse of products as complements for the improvement of construction materials in order to generate a sustainable construction that is friendly with the environment, as proposed by the circular economy which is a concept related to the use of resources. A linear economy proposes the use of resources from the beginning to the end, while the circular one tries to reuse them by inserting them back into the process, this could also be understood as the optimization of the life cycle. This article describes the evaluation of technological components used in the mixture for the elaboration of building clay blocks, such as tree leaves and palm rachis ash, with the objective of estimating their feasibility in costs and making a diagnosis of the different properties that these components provide to the mix, as well as the requirements that clay blocks must meet according to the Colombian earthquake-resistant standard NSR10.

Keywords: evaluation, reuse, clay blocks, feasibility, environment, diagnosis. 
Introducción

El sector de la construcción es considerado mundialmente una de las principales fuentes de contaminación ambiental que existen, pues genera impactos negativos directa 0 indirectamente en este, por su naturaleza la construcción no es un proceso amigable con el medio ambiente [1] puesto que son muy pocos los contratistas o empresas privadas que invierten sus esfuerzos en desarrollar una política de reutilización o reciclaje de sus residuos sólidos [2]. Teniendo en cuenta el gran número de construcciones que se realizan a diario en el mundo, el impacto sobre el ecosistema se ha convertido en asunto de vital importancia [3].

De esta manera, se habla de componentes adversos como: polvo, desechos, generación de tóxicos, contaminación del aire y agua, cambio climático, malos olores, uso del suelo, intervención con maquinaria para remover vegetación y emisiones peligrosas [4]. Actualmente países como Brasil genera cantidades de residuos y produce grandes cantidades de consumo, debido a factores tales como la acelerada expansión económica o a los incentivos por parte del gobierno [5], y la inversión en las infraestructuras que se precisan para celebrar eventos de talla mundial como la Copa del Mundo y las Olimpiadas, que de acuerdo con datos del Conselho Brasileiro de Construção Sustentável (2011), la construcción y manutención de infraestructuras en este país consume cerca de $75 \%$ de los recursos naturales y el uso de los edificios es responsable de más del $45 \%$ del consumo de energía eléctrica, valor que crece más rápido que la economía del país.

Así mismo, en Colombia una de las fuentes contaminantes también es la industria de la construcción, la cual tiene un alto impacto en el desarrollo económico y social de cualquier región y se enmarca en el modelo de crecimiento [6]. De acuerdo con el Departamento Administrativo Nacional de Estadística (DANE), en el cuarto trimestre del 2016 el valor agregado de la construcción creció $4.1 \%$ con respecto al año anterior, esto debido al incremento de la construcción de edificaciones como viviendas de interés social (VIS) en 6,0\% y en obras civiles como nuevas vías o reparación y mantenimiento de viejas edificaciones al $2,4 \%$, trayendo consigo impactos ambientales negativos tales como los mencionados anteriormente.

En este contexto, los gremios constructores del país tienen la responsabilidad de cumplir un papel vital: correctivo, preventivo y pedagógico, basados en el panorama actual del medio ambiente, minimizando los impactos destructivos en este, ahondando en nuevas practicas constructivas como la creación de programas de reciclaje y reutilización de residuos sólidos que permitan afrontar esta grave situación.

Por lo anterior, uno de los grandes retos para la Ciencia e Ingeniería, es el desarrollo de materiales con criterios o parámetros de sostenibilidad ambiental. Los ladrillos son uno de los elementos de mampostería más utilizados en la construcción [7] y es por esto que lo convierte en el componente clave para realizar ese cambio significativo que tanto se requiere, utilizando nutrientes orgánicos en la mezcla de su fabricación tales como hojas de árboles o ceniza de raquis de palma, con el propósito de mejorar sus propiedades y disminuir la extracción de la materia prima como lo es la arcilla, minimizando los impactos geológicos que esta ocasiona al medio ambiente [8].

Por ejemplo, en estudios realizados anteriormente se ha demostrado que sustituyendo un $15 \%$ de cemento, por arcilla en un $10 \%$ y cenizas de hoja de schinus (MOLLE) en un $5 \%$ se ha logrado alcanzar una resistencia 
a la comprensión considerablemente similar a la de un ladrillo fabricado con una mezcla común [9] y de este mismo modo, se comprobó que el uso de la fibra del raquis de palma africana en la fabricación de ladrillos para fines de construcción es posible, ya que mejoran las propiedades físicas y mecánicas del ladrillo convencional, además de disminuir costos de producción y el impacto negativo al ambiente [10], demostrando así que el uso de estos nutrientes tecnológicos en la mezcla de fabricación de ladrillos de arcilla podría ser una posible solución a los problemas medioambientales mencionados con anterioridad.

\section{Fundamentación Teórica}

La actividad minera es una de las que genera mayores ingresos económicos en Colombia, gracias a la diversidad de minerales que están presentes en el territorio nacional [11], no obstante, en la actualidad se vienen desarrollando en la región una serie de conflictos socio-ambientales por causa de la explotación de los recursos naturales. Esto se debe en concreto a las prácticas de extracción ilegal de los minerales $o$ al incumplimiento de las obligaciones ambientales y sociales derivadas de la legislación y de los instrumentos ambientales [12]. Sin embargo, hasta el momento se ha visto como diferentes residuos de materias orgánicas han sido estudiados para reemplazar cantidades de arcilla en la producción de ladrillos para la construcción.

Por una parte, se ha estudiado la composición en función de las distintas partes de los árboles como ramas y raíces, las cuales son más ricas en elementos como el P, K, y Mg que el tronco, la corteza y las acículas. [13] Sin embargo, los mayores valores de $\mathrm{Ca}, \mathrm{Mn}$ Al y $\mathrm{S}$ fueron las que provenían de las cenizas de corteza. Por otra parte, cómo la concentración de $\mathrm{Ca}, \mathrm{Mg}$ y Fe aumentan con la edad de las hojas [14] y el
$\mathrm{N}, \mathrm{P}$ y K disminuyen, su concentración en las cenizas también, las cuales en su proceso de combustión generan, por ejemplo, cenizas de fondoocenizas volantes, ocenizas deresiduos, demostrándose en diferentes estudios que estas últimas presentan gran variabilidad en la concentración de macronutrientes [15].

De la misma manera, el raquis de palma africana es un residuo del proceso industrial de extracción de aceite y que constituye aproximadamente el $50 \%$ en peso de la fruta para procesar. Esto quiere decir que existe una gran disponibilidad del material que en la práctica es utilizado como fuente de energía en grandes extractoras y como barreras contra insectos en palma jóvenes. [10] Conteniendo notables propiedades de resistencia, absorción de humedad, flexibilidad y esbeltez en la fabricación de bloques de arcilla para construcción de edificaciones, dando oportunidad a un producto menos contaminante e innovador [16].

\section{Materiales y métodos}

En el siguiente artículo se emplea un tipo de investigación descriptiva y documental, pues se busca realizar una evaluación de datos provenientes de investigaciones elaboradas con nutrientes tecnológicos adicionados a la mezcla de arcilla para la fabricación de ladrillos de construcción, este tipo de investigación consiste en la recopilación de datos que describen los acontecimientos y luego organiza, tabula, representa y describe dicha recopilación [17]. De esta manera, se estimará si las propiedades que estos nutrientes aportan a la mezcla mejoran su composición y cuanto sería el costo aproximado que esta tendría.

En este orden de ideas, los artículos o investigaciones correspondientes a evaluar son la tesis de ingeniería civil titulada: 


\section{4}

"Resistencia a la Compresión de Ladrillo de Concreto, Sustituyendo un $15 \%$ al Cemento, por Arcilla en un $10 \%$ y Cenizas de Hoja de Schinus (MOLLE) en un 5\%" de la universidad de San Pedro en Perú, realizada por el ingeniero Anthony Richard Obregón Cruz, y la tesis en ingeniería en biotecnología ambiental titulada: "Factibilidad del Uso del Raquis de Palma Africana en Mezcla con Agregados de Construcción para la Fabricación de Ladrillos Ecológicos" de la escuela superior politécnica de Chimborazo en Ecuador, realizada por la ingeniera Daniela Cecilia Páliz Hidalgo.

Para la elaboración del instrumento de recolección de información se llevó a cabo una separación de los datos y un análisis comparativo entre estas dos investigaciones, organizando los resultados de estas que pudieran servir para llevar a cabo los objetivos de este artículo. De igual forma, se efectuó un estudio detallado del proceso de realización de estas para comprender de una mejor manera como los nutrientes tecnológicos usados en las muestras mejoran la mezcla de arcilla y en este sentido verificar si se cumple con los requisitos exigidos por la norma sismorresistente colombiana NSR10.

\section{Resultados y Discusión}

Los ladrillos ecológicos constituyen insumos potenciales para el desarrollo y mejora en la calidad de los elementos de construcción de viviendas, en especial para las familias de escasos recursos económicos, empleando tecnologías y materiales que disminuyan el impacto ambiental, ya que para su fabricación se requiere de un gasto mínimo de energía (necesitan tan sólo una temperatura de cocción de $60^{\circ} \mathrm{C}$ ), se reducen en un $85 \%$ las emisiones de gases de efecto invernadero, y otros son secados al ambiente, por tanto se reducen las emisiones de gases de dióxido de carbono a la atmósfera, consumo de materia prima que requieren los elementos de construcción convencionales de procesamiento sencillo, mejores aislantes del frío y del calor exterior, consumen menos energía, son más económicos, más ligeros y manejables para el trabajador, agilizando el tiempo de construcción y disminuyendo el gasto de materiales.

Resistencia a la compresión de ladrillo de concreto sustituyendo un $15 \%$ al cemento, por arcilla en un $10 \%$ y cenizas de hoja de Schinus (Mole) en un 5\%

En cuanto a los datos de la primera investigación: "Resistencia a la Compresión de Ladrillo de Concreto, Sustituyendo un $15 \%$ al Cemento, por Arcilla en un 10\% y Cenizas de Hoja de Schinus (MOLLE) en un 5\%", se tomaron muestras de arcilla extraídas de la localidad de Carhuaz en Perú, su componente principal es el óxido de calcio, responsable de su alta reactividad, con el que se busca una alternativa del recurso para el diseño de un ladrillo de concreto de alta resistencia. Así mismo, en la ciudad de Huaraz los desechos sólidos ocupan un gran espacio y causan problemas de salud e impacto en el medio ambiente y algunos de estos desechos son de origen vegetal como las hojas y ramas de árboles y plantas, debido a su descomposición.

Tal como las muestras de arcillas, estos desechos sólidos tienen como ingrediente principal el dióxido de silicio, responsable de su alta reactividad, se observará si es un remplazo porcentual en peso del cemento de la mezcla convencional y si los resultados obtenidos son favorables para considerarse a la ceniza de hojas de schinus (molle) una opción de reemplazo en la mezcla de fabricación de ladrillos de concreto convencionales.

El método de investigación utilizado en esta tesis de grado es de experimentación, con un tipo o enfoque aplicado ya que se busca resolver una problemática conocida y encontrar respuestas a preguntas específicas, 
Andrés Felipe Ruíz, Carlos Javier Peñaranda,

55

usando métodos innovadores con materiales de bajo costo para generar unidades de mampostería que permitan diseñar viviendas con mayor durabilidad y mejor resistencia, evaluando dos muestras respectivamente seleccionadas mediante un ensayo, donde se obtendrían los resultados de dos grupos de estudios que se denominaron: Grupo Control y Grupo Experimental, utilizando los materiales mencionados anteriormente sustituyéndolos porcentualmente entre los elementos que conforma la mezcla para elaborar un ladrillo de concreto convencional.

Una vez obtenidos todos los materiales para la elaboración de la mezcla, se llevó a cabo el lavado y secado de la arcilla así como su calcinación, el pre quemado de las hojas de schinus (molle) durante un periodo de 20 días al aire libre, para posteriormente realizar la calcinación de estas en una mufla (horno) a una temperatura de $900^{\circ} \mathrm{c}$, se tomó la cantidad de material para tres ladrillos de acuerdo al diseño de mezcla y luego se procedió a pesar los porcentajes para la sustitución del cemento, arcilla el $10 \%$ del cemento y cenizas de hoja de schinus (molle) en un 5\% mezclándose manualmente.

De esta manera, se continuo con la fabricación de 18 ladrillos patrón realizando el vaciado de cada uno de manera instantánea llenando todos los moldes, para luego desencofrarlos y realizar el respectivo curado por un periodo de tiempo de 7, 14 y 28 días y por último evaluar su resistencia a la compresión. Del mismo modo se elaboró la misma cantidad de ladrillos con la mezcla experimental y una resistencia de concreto de f'c $=130 \mathrm{~kg} / \mathrm{cm} 2$, mezclando los materiales secos de manera manual y agregando agua para formar una mezcla moldeable y manejable, se realizó el vaciado de cada ladrillo en forma continua llenando así los ladrillos con dicha mezcla.
Tabla 1. Pesos de los agregados por la cantidad de ladrillos a elaborar

\begin{tabular}{lccccr}
\hline \multicolumn{3}{c}{ PESOS } & & \\
\hline & PESO & \multicolumn{2}{c}{ CANTIDAD } & & $\begin{array}{c}\text { TOTAL DE } \\
\text { LADRILLOS }\end{array}$ \\
\hline EN KG & & & & 8.046 \\
Cemento & 0.894 & $\mathrm{x}$ & 9 & $=$ & 34.389 \\
Agregado Fino & 3.821 & $\mathrm{x}$ & 9 & $=$ & 21.663 \\
Agua & 2.407 & $\mathrm{x}$ & 9 & $=$ & 7.56 \\
\hline
\end{tabular}

Fuente: Resistencia a la Compresión de Ladrillo de Concreto, Sustituyendo un $15 \%$ al Cemento, por Arcilla en un $10 \%$ y Cenizas de Hoja de Schinus (MOLLE) en un $5 \%$.

Tabla 2. Pesos de la materia prima en porcentajes para la sustitución del cemento.

\begin{tabular}{lcc}
\hline \multicolumn{3}{c}{ PESO POR UNIDAD } \\
\hline \multicolumn{1}{c}{ MATERIA PRIMA } & PORCENTAJE & PESO EN (kg) \\
Arcilla & $10 \%$ & 0.089 \\
CHS (molle) & $5 \%$ & 0.045 \\
Total de la Sustitución $\%$ & $15 \%$ & 0.134 \\
Cemento & $85 \%$ & 0.760 \\
Combinación & $100 \%$ & 0.894 \\
\hline
\end{tabular}

Fuente: Resistencia a la Compresión de Ladrillo de Concreto, Sustituyendo un $15 \%$ al Cemento, por Arcilla en un $10 \%$ y Cenizas de Hoja de Schinus (MOLLE) en un $5 \%$.

Tabla 3. Resultados del ensayo de compresión del concreto patrón a los 7 días de curado

\begin{tabular}{|c|c|c|c|c|c|c|c|c|}
\hline \multirow{2}{*}{$\begin{array}{l}\text { LADRILLO } \\
\text { PATRON }\end{array}$} & \multicolumn{3}{|c|}{$\begin{array}{l}\text { MEDIDAS DEL } \\
\text { LADRILLO }\end{array}$} & \multirow{2}{*}{$\begin{array}{c}\text { AREA } \\
\text { BRUTA } \\
(\mathrm{cm} 3)\end{array}$} & \multirow{2}{*}{$\begin{array}{c}\text { CARGA } \\
\text { DE } \\
\text { ROTURA } \\
\text { (kg) }\end{array}$} & \multirow{2}{*}{$\begin{array}{c}\text { fo } \\
\text { (KG/CM2) }\end{array}$} & \multirow{2}{*}{$\begin{array}{c}\text { fo } \\
\operatorname{prom}(\mathbf{K G} / \mathrm{CM} 2)\end{array}$} & \multirow{2}{*}{$\%$} \\
\hline & LARGO & ANCHO & ALTO & & & & & \\
\hline P1 & 23.97 & 13.95 & 9.00 & 334.38 & 36370 & 108.77 & \multirow{3}{*}{105.46} & \multirow{3}{*}{81.12} \\
\hline $\mathrm{P} 2$ & 23.95 & 13.95 & 9.00 & 334.10 & 34980 & 104.70 & & \\
\hline P3 & 23.95 & 13.95 & 9.00 & 334.10 & 34380 & 102.90 & & \\
\hline
\end{tabular}

Fuente: Resistencia a la Compresión de Ladrillo de Concreto, Sustituyendo un $15 \%$ al Cemento, por Arcilla en un $10 \%$ y Cenizas de Hoja de Schinus (MOLLE) en un $5 \%$.

Tabla 4. Resultados del ensayo de compresión del concreto patrón a los 14

\begin{tabular}{|c|c|c|c|c|c|c|c|c|}
\hline \multirow{2}{*}{$\begin{array}{l}\text { LADRILLO } \\
\text { PATRON }\end{array}$} & \multicolumn{3}{|c|}{$\begin{array}{c}\text { MEDIDAS DEL } \\
\text { LADRILLO }\end{array}$} & \multirow{2}{*}{$\begin{array}{c}\text { AREA } \\
\text { BRUTA } \\
(\mathbf{c m} 3) \\
\end{array}$} & \multirow{2}{*}{$\begin{array}{c}\text { CARGA } \\
\text { DE } \\
\text { ROTURA } \\
(\mathrm{kg}) \\
\end{array}$} & \multirow{2}{*}{$\begin{array}{c}\text { fo } \\
\text { (KG/CM2) } \\
\end{array}$} & \multirow{2}{*}{$\begin{array}{c}\text { fo } \\
\operatorname{prom}(\mathbf{K} G / \mathrm{CM} 2)\end{array}$} & \multirow{2}{*}{$\%$} \\
\hline & LARGO & ANCHO & ALTO & & & & & \\
\hline P4 & 23.97 & 13.98 & 8.98 & 335.10 & 39450 & 117.73 & \multirow{3}{*}{122.72} & \multirow{3}{*}{94.40} \\
\hline P5 & 23.98 & 13.98 & 8.97 & 335.24 & 42840 & 127.79 & & \\
\hline P6 & 24.00 & 14.00 & 9.00 & 336.00 & 41210 & 122.65 & & \\
\hline
\end{tabular}

Fuente: Resistencia a la Compresión de Ladrillo de Concreto, Sustituyendo un $15 \%$ al Cemento, por Arcilla en un $10 \%$ y Cenizas de Hoja de Schinus (MOLLE) en un 5\%. 
Tabla 5. Resultados del ensayo de compresión del concreto patrón a los 28 días de curado

\begin{tabular}{|c|c|c|c|c|c|c|c|c|}
\hline \multirow{2}{*}{$\begin{array}{l}\text { LADRILLO } \\
\text { PATRON }\end{array}$} & \multicolumn{3}{|c|}{$\begin{array}{l}\text { MEDIDAS DEL } \\
\text { LADRILLO }\end{array}$} & \multirow{2}{*}{$\begin{array}{c}\text { AREA } \\
\text { BRUTA } \\
(\mathbf{c m} 3)\end{array}$} & \multirow{2}{*}{$\begin{array}{c}\text { CARGA } \\
\text { DE } \\
\text { ROTURA } \\
\text { (kg) }\end{array}$} & \multirow{2}{*}{$\begin{array}{c}\text { fo } \\
\text { (KG/CM2) }\end{array}$} & \multirow{2}{*}{$\begin{array}{c}\text { fo } \\
\operatorname{prom}(\text { KG/CM2) }\end{array}$} & \multirow{2}{*}{$\%$} \\
\hline & LARGO & ANCHO & ALTO & & & & & \\
\hline P7 & 23.93 & 13.97 & 8.93 & 334.30 & 42760 & 127.91 & \multirow{3}{*}{131.63} & \multirow{3}{*}{101.25} \\
\hline P8 & 24.00 & 13.83 & 8.94 & 331.92 & 46250 & 139.34 & & \\
\hline P9 & 23.92 & 13.97 & 8.98 & 334.16 & 42650 & 127.63 & & \\
\hline
\end{tabular}

Fuente: Resistencia a la Compresión de Ladrillo de Concreto, Sustituyendo un $15 \%$ al Cemento, por Arcilla en un $10 \%$ y Cenizas de Hoja de Schinus (MOLLE) en un $5 \%$.

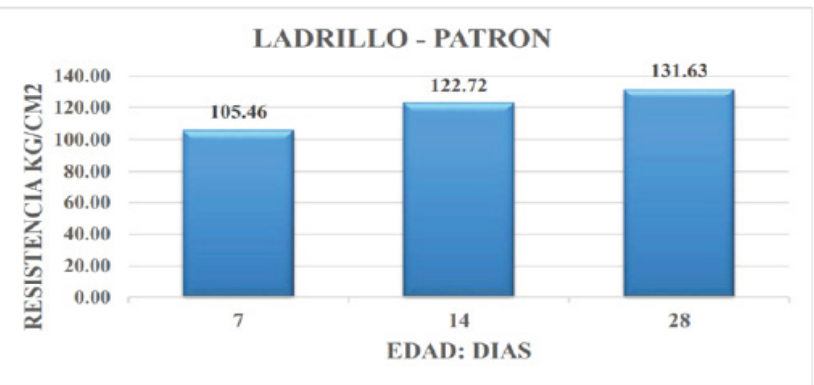

Grafica 1. Resistencia a la compresión de ladrillo - Patrón

Fuente: Resistencia a la Compresión de Ladrillo de Concreto, Sustituyendo un $15 \%$ al Cemento, por Arcilla en un $10 \%$ y Cenizas de Hoja de Schinus (MOLLE) en un $5 \%$.

Tabla 6. Resultados del ensayo de compresión del concreto patrón con mezcla experimental a los 7 días de curado

\begin{tabular}{|c|c|c|c|c|c|c|c|c|}
\hline \multirow{2}{*}{$\begin{array}{l}\text { LADRILLO } \\
\text { PATRON }\end{array}$} & \multicolumn{3}{|c|}{$\begin{array}{l}\text { MEDIDAS DEL } \\
\text { LADRILLO }\end{array}$} & \multirow{2}{*}{$\begin{array}{c}\text { AREA } \\
\text { BRUTA } \\
(\mathrm{cm} 3)\end{array}$} & \multirow{2}{*}{$\begin{array}{c}\text { CARGA } \\
\text { DE } \\
\text { ROTURA } \\
\text { (kg) }\end{array}$} & \multirow{2}{*}{$\begin{array}{c}\mathrm{fo}_{0} \\
\text { (KG/CM2) }\end{array}$} & \multirow{2}{*}{$\begin{array}{c}\mathrm{fo}_{0} \\
\operatorname{prom}(\mathbf{K G} / \mathrm{CM} 2)\end{array}$} & \multirow{2}{*}{$\%$} \\
\hline & LARGO & АNCHO & ALTO & & & & & \\
\hline E1 & 23.95 & 13.97 & 8.93 & 334.6 & 32070 & 95.85 & \multirow{3}{*}{97.97} & \multirow{3}{*}{75.36} \\
\hline E2 & 23.83 & 13.93 & 8.95 & 331.0 & 33470 & 99.61 & & \\
\hline E3 & 23.83 & 13.90 & 9.00 & 334.2 & 32610 & 98.45 & & \\
\hline
\end{tabular}

Fuente: Resistencia a la Compresión de Ladrillo de Concreto, Sustituyendo un $15 \%$ al Cemento, por Arcilla en un $10 \%$ y Cenizas de Hoja de Schinus (MOLLE) en un $5 \%$.

Tabla 7. Resultados del ensayo de compresión del concreto patrón con mezcla experimental a los 14 días de curado

\begin{tabular}{|c|c|c|c|c|c|c|c|c|}
\hline \multirow{2}{*}{$\begin{array}{l}\text { LADRILLO } \\
\text { PATRON }\end{array}$} & \multicolumn{3}{|c|}{$\begin{array}{l}\text { MEDIDAS DEL } \\
\text { LADRILLO }\end{array}$} & \multirow{2}{*}{$\begin{array}{c}\text { AREA } \\
\text { BRUTA } \\
(\mathrm{cm} 3)\end{array}$} & \multirow{2}{*}{$\begin{array}{c}\text { CARGA } \\
\text { DE } \\
\text { ROTURA } \\
(\mathrm{kg}) \\
\end{array}$} & \multirow{2}{*}{$\begin{array}{c}\text { fo } \\
\text { (KG/CM2) }\end{array}$} & \multirow{2}{*}{$\begin{array}{c}\text { fo } \\
\operatorname{prom}(\mathrm{KG} / \mathrm{CM} 2)\end{array}$} & \multirow{2}{*}{$\%$} \\
\hline & LARG0 & ANCHO & ALTO & & & & & \\
\hline E4 & 23.97 & 13.93 & 8.95 & 333.9 & 38870 & 116.41 & \multirow{3}{*}{118.02} & \multirow{3}{*}{90.79} \\
\hline E5 & 23.90 & 13.98 & 9.00 & 334.1 & 39980 & 119.66 & & \\
\hline E6 & 23.93 & 13.90 & 9.83 & 332.6 & 39250 & 118,02 & & \\
\hline
\end{tabular}

Fuente: Resistencia a la Compresión de Ladrillo de Concreto, Sustituyendo un $15 \%$ al Cemento, por Arcilla en un $10 \%$ y Cenizas de Hoja de Schinus (MOLLE) en un $5 \%$.
Tabla 8. Resultados del ensayo de compresión del concreto patrón con mezcla experimental a los 28 días de curado

\begin{tabular}{|c|c|c|c|c|c|c|c|c|}
\hline \multirow{2}{*}{$\begin{array}{l}\text { LADRILLO } \\
\text { PATRON }\end{array}$} & \multicolumn{3}{|c|}{$\begin{array}{l}\text { MEDIDAS DEL } \\
\text { LADRILLO }\end{array}$} & \multirow{2}{*}{$\begin{array}{r}\text { AREA } \\
- \text { BRUTA } \\
(\mathrm{cm} 3)\end{array}$} & \multirow{2}{*}{$\begin{array}{c}\text { CARGA } \\
\text { DE } \\
\text { ROTURA } \\
\text { (kg) }\end{array}$} & \multirow{2}{*}{$\begin{array}{c}\text { fo } \\
\text { (KG/CM2) }\end{array}$} & \multirow{2}{*}{$\begin{array}{c}\text { fo } \\
\operatorname{prom}(\mathrm{KG} / \mathrm{CM} 2)\end{array}$} & \multirow{2}{*}{$\%$} \\
\hline & LARGO & ANCHO & ALTO & & & & & \\
\hline E7 & 23.97 & 13.87 & 8.97 & 332.5 & 42900 & 129.04 & \multirow{3}{*}{125.50} & \multirow{3}{*}{96.54} \\
\hline E8 & 23.93 & 13.92 & 8.98 & 333.1 & 42670 & 128.10 & & \\
\hline E9 & 23.90 & 13.93 & 8.93 & 332.9 & 39740 & 119.37 & & \\
\hline
\end{tabular}

Fuente: Resistencia a la Compresión de Ladrillo de Concreto, Sustituyendo un $15 \%$ al Cemento, por Arcilla en un $10 \%$ y Cenizas de Hoja de Schinus (MOLLE) en un $5 \%$.

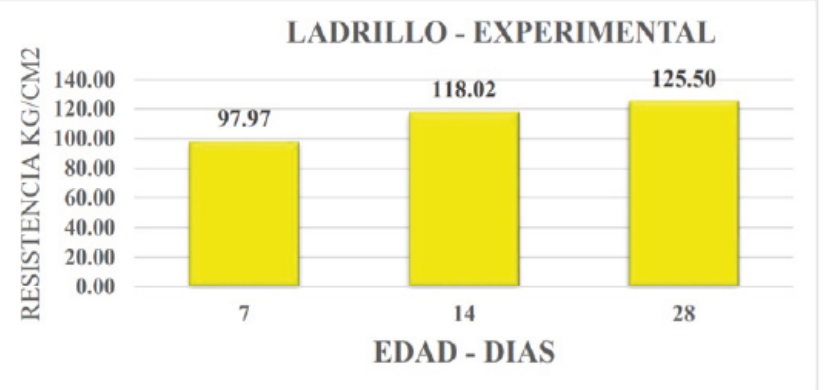

Grafica 2. Resistencia a la compresión de ladrillo - Patrón

Fuente: Resistencia a la Compresión de Ladrillo de Concreto, Sustituyendo un $15 \%$ al Cemento, por Arcilla en un $10 \%$ y Cenizas de Hoja de Schinus (MOLLE) en un $5 \%$.

Los resultados obtenidos en la elaboración de los ladrillos no fueron favorables, puesto que los ladrillos experimentales elaborados por la sustitución del cemento en un $15 \%$, por un $10 \%$ de arcilla y ceniza de hoja de schinus (molle) en un $5 \%$, alcanzo una resistencia promedio de $125.50 \mathrm{~kg} / \mathrm{cm} 2$ a la edad de 28 días, con un porcentaje de $96.54 \%$, por lo cual no supero al ladrillo patrón ya que su resistencia promedio a los 28 días es de $131.63 \mathrm{~kg} / \mathrm{cm} 2$ con un porcentaje de $101.25 \%$ de tal manera se destaca que esta investigación no agota ni frena el tema, solo da una importante apertura al desarrollo de materiales alternativos de construcción.

Factibilidad del uso del Raquis de Palma Africana en mezcla con agregados de construcción para la fabricación de ladrillos ecológicos

Para esta investigación se revalorizó el "raquis" residuo de palma africana, usándolo 
como nutriente tecnológico en la elaboración de ladrillos ecológicos que posteriormente serán utilizados en la construcción, realizando análisis físico-químicos para conocer las características y composición elemental del residuo, formulando cuatro mezclas diferentes una vez caracterizada la fibra, la primera mezcla formada por $38 \%$ de raquis, $18 \%$ de arena, $32 \%$ de arcilla y $32 \%$ cemento; la segunda mezcla $41 \%$ de raquis y $50 \%$ de arcilla; la tercera mezcla $51 \%$ de raquis, $18 \%$ de arena y $5 \%$ de cemento; y la cuarta mezcla formada por $58 \%$ de raquis y $34 \%$ de cemento. Finalmente, para conocer las características mecánicas y físicas de los ladrillos fabricados, se realizaron pruebas de compresión, flexión y humedad.

El método de investigación empleado en esta tesis de grado es de aplicación, pues se busca determinar la factibilidad del uso del raquis en mezcla con agregados de construcción para la fabricación de los ladrillos ecológicos, realizando la recolección de la materia prima en sacas o bolsas de tela y de forma manual, recolectando entre 10 y 15 raquis para posteriormente situarlo al aire libre en presencia del sol para su secado por un tiempo de un mes. El proceso de molienda y triturado se hizo a través de una banda transportadora que dirige el material hasta un molino mecánico, donde se le extrae el aceite que este pueda contener y al finalizar el proceso pasa de ser una estructura húmeda y pesada, a ser, una fibra suave y con poco contenido de humedad.

Una vez listo el material para ser usado, se procedió a diseñar los cuatro ladrillos ecológicos con su respectiva materia prima y formulaciones. La forma de los modelos del Ladrillo ecológico es de un cubo rectangular, macizo, pueden tener imperfecciones en sus caras exteriores, así como variaciones de rectitud en sus aristas, por ser fabricados a mano, con unas medidas de $25 \mathrm{~cm}$ de largo,
$12 \mathrm{~cm}$ de ancho y $5 \mathrm{~cm}$ de alto. El raquis es considerado como una fibra con alto contenido de humedad por lo que es utilizado como fuente de hidratación para las palmeras en épocas de mucho sol, para conocer el contenido de humedad de la fibra de Raquis, se tomaron tres muestras de la misma y se calcularon sus porcentajes de humedad.

Tabla 9. Resultados de contenido de humedad en la fibra

\begin{tabular}{|c|c|c|c|c|c|}
\hline $\begin{array}{c}\text { FIB RA } \\
\text { VEGETAL }\end{array}$ & $\begin{array}{c}\text { Cantidad } \\
\text { de la } \\
\text { muestra (g) }\end{array}$ & $\begin{array}{c}\text { Peso deI } \\
\text { plato vacio } \\
\text { (g) }\end{array}$ & $\begin{array}{c}\text { Plato + } \\
\text { muestra } \\
\text { húmeda (g) }\end{array}$ & $\begin{array}{c}\text { Plato }+ \\
\text { muestra } \\
\text { seca (g) }\end{array}$ & $\begin{array}{c}\text { \% de } \\
\text { Humedad }\end{array}$ \\
\hline MUESTRA 1 & 5 & 3,67 & 8,67 & 7,45 & 24 \\
\hline MUESTRA 2 & 5 & 0,71 & 5,71 & 4,11 & 32 \\
\hline MUESTRA 3 & 5 & 0,75 & 5,75 & 3,55 & 44 \\
\hline \multicolumn{5}{|c|}{ PROMEDIO } \\
\hline
\end{tabular}

Fuente: Factibilidad del Uso del Raquis de Palma Africana en Mezcla con Agregados de Construcción para la Fabricación de Ladrillos Ecológicos

De acuerdo a los resultados obtenidos en la prueba de compresión con una edad de 21 días se tuvo que el primer ladrillo ecológico Modelo "A" presentó una resistencia de 14,38 $\mathrm{Mpa}$, el segundo ladrillo Modelo "B" 4,4 Mpa, el tercer ladrillo Modelo "C" 3, 34 Mpa y el cuarto ladrillo Modelo "D" 11,20 Mpa. Los Modelos A y D superan los valores de resistencia a la compresión con respecto a lo establecido en la Norma Ecuatoriana INEN 297, "Ladrillos Cerámicos, Requisitos" la misma que reporta un valor de 6 Mpa como requisito, de la misma manera, estos valores cumplen con lo establecido en la Norma Sismorresistente Colombiana NSR10 que en su título D.7.1.4. especifica que la resistencia a la compresión de la mampostería elaborada con arcilla y perforaciones verticales no puede tener una resistencia menor de $10 \mathrm{Mpa}$. Por lo tanto, el raquis ayuda a mantener la forma del ladrillo mientras recibe el peso para su deformación en la presente prueba.

Por otra parte, los ladrillos B y C no superaron los valores establecidos en la norma. El ladrillo $\mathrm{B}$ cuya composición es arcilla y raquis para haber obtenido mayor resistencia y durabilidad 


\section{8}

tendría que haber sido horneado como un ladrillo convencional, debido a que la arcilla necesita entrar en un proceso de cocción para perder su elasticidad y volverse dura y compacta, pero no fue así, en la presente investigación su secado se realizó al aire libre, que no fue suficiente para obtener un ladrillo resistente en esta prueba. $\mathrm{Y}$ en el ladrillo $\mathrm{C}$ el raquis por encontrarse en mayor cantidad absorbió gran parte de los componentes dejando un ladrillo delgado y frágil lo que no permitió resistir la prueba.

Una vez realizado los análisis y las respectivas pruebas, se obtuvo que el Ladrillo Ecológico apto y calificado para la presente investigación sea el Ladrillo Modelo "A", para el fin al que se le quiera destinar ya sea en construcción mampostería liviana u obras civiles, su resistencia y capacidad de absorción son los dos puntos que tuvieron mayor relevancia. Otro aspecto que se tomó en cuenta es su peso, el ladrillo Modelo "A" es más liviano que un ladrillo convencional, su peso fue de 1,07 $\mathrm{grs} / \mathrm{cm} 3$ mientras que el ladrillo convencional tiene un peso de 1,80 grs/cm3. En la Tabla 10 se muestra cada una de las características e información adicional del ladrillo "A" y demás ladrillos fabricados.

Tabla 10. Características de los ladrillos fabricados

\begin{tabular}{|c|c|c|c|c|}
\cline { 2 - 5 } \multicolumn{1}{c|}{} & \multicolumn{4}{c|}{ MUESTRA (4) } \\
\cline { 2 - 5 } \multicolumn{1}{c|}{} & A & B & C & D \\
\hline $\begin{array}{c}\text { Fecha de } \\
\text { fabricación }\end{array}$ & 28 -ene-15 & 28 -ene-15 & 28 -ene-15 & 28 -ene-15 \\
\hline Fecha de rotura & 18 -feb-15 & 18 -feb-15 & 18 -feb-15 & 18 -feb-15 \\
\hline Edad (dias) & 21 & 21 & 21 & 21 \\
\hline Masa (gr.) & 80,4 & 102,6 & 54 & 101 \\
\hline $\begin{array}{c}\text { Area de contacto } \\
\text { (cm) }\end{array}$ & 25 & 25 & 25 & 25 \\
\hline Espesor (cm) & 3 & 3 & 3 & 3 \\
\hline Volumen (cm3) & 75 & 75 & 75 & 75 \\
\hline $\begin{array}{c}\text { PESO } \\
\text { UNITARIO } \\
\text { (grs./cm3) }\end{array}$ & 1,07 & 1,37 & 0,72 & 1,35 \\
\hline
\end{tabular}

Fuente: Factibilidad del Uso del Raquis de Palma Africana en Mezcla con Agregados de Construcción para la Fabricación de Ladrillos Ecológicos
Factibilidad de los costos

El ladrillo es producido de dos maneras, la primera artesanalmente, que utilizan medios muy rudimentarios para la elaboración el cual necesita ser cocido antes de salir al mercado para que obtenga resistencia, [18] este producto artesanal tiene muchas fallas de molde lo que provoca al momento de ser utilizado en el levantamiento de paredes y en el enlucido que se absorba mucho cemento el cual ocasiona un aumento de costos. De igual manera el ladrillo elaborado en forma industrial, no utilizan mayor tecnología para la elaboración, el producto tiene que ser cocido en hornos para llevarlo al mercado. Este proceso de cocido hace que los costos sean más altos. El incremento de los precios de los materiales de construcción, ocasiona que se eleve el costo de producción y afecta la oferta de viviendas y demás tipos de obras de construcción [19].

Ante esta situación, no existen en el mercado alternativas más económicas en materiales de construcción que igualen o superen los beneficios y calidad de los materiales ya existentes. La necesidad de establecer variables fundamentales como el costo de fabricación de ladrillos hechos con nutrientes tecnológicos que provengan de materiales orgánicos y reciclables, conlleva a realizar este artículo que sirva de ayuda para despejar las dudas sobre el valor que tendría el uso de la maquinaria, conseguir la materia prima, gastos operativos, no operativos, la cantidad que se podría producir según las especificaciones técnicas de la maquinaria y su depreciación final. El cuadro siguiente desglosa los rubros que se ocuparían al fabricar el ladrillo industrial convencional en el periodo de un año. 
Tabla 11. Gasto y Costos para precio referencial del Ladrillo

\begin{tabular}{lcc}
\hline \multicolumn{1}{c}{ PRECIO } & \\
\hline \multicolumn{1}{c}{ DETALLE } & COSTOS \\
\hline Costos Directos & FIJOS & VARIABLES \\
\hline Costos Indirectos & $45.676,14$ \\
\hline Insumos & $18.909,60$ \\
\hline Reparación y Mantenimiento & $11.130,80$ \\
\hline Amortización & $11.843,96$ \\
\hline Depreciación & $19.461,06$ \\
\hline Subtotales & $19.970,30$ \\
\hline Total de Costos de Producción & $58.340,96$ \\
\hline Gasto de alquiler de local para oficina & $97.021,56$ \\
\hline Gasto de alquiler de local para fabrica & $14.400,00$ \\
\hline Gasto de Servicios Básicos & $36.000,00$ \\
\hline Gasto de suministro de oficina & $12.720,00$ \\
\hline Gasto de Suministros de limpieza & 850,00 \\
\hline Gasto de Combustible & 840,00 \\
\hline Gastos de sueldos & $14.400,00$ \\
\hline Gasto de publicidad & $56.276,70$ \\
\hline Gasto en Promoción & $38.900,00$ \\
\hline Subtotales & 672,00 \\
\hline Costo Total & $175.058,70$ \\
\hline Unidades de Ladrillos & $272.080,26$ \\
\hline Costo Unitario de Ladrillo & $1.141 .903,53$ \\
\hline UTILIDAD 10\% & 0,24 \\
\hline PRECIO NETO & 0,04 \\
\hline
\end{tabular}

Fuente: Proyecto de factibilidad para la fabricación de ladrillos, con polietileno de alta densidad $100 \%$ reciclado.

Tabla 12. Balance de Maquinaria y Equipo necesario para la fabricación de los ladrillos

\begin{tabular}{|c|c|c|c|c|}
\hline Descripción & Cant. & & costos (en USD) & $\begin{array}{c}\text { Tiempo } \\
\text { de } \\
\text { Vida/Años }\end{array}$ \\
\hline Triturador & 1 & $\$$ & $10.710,00$ & 10 \\
\hline Mezcladora & 1 & $\$$ & $10.914,00$ & 10 \\
\hline Banda Transportadora & 1 & $\$$ & $1.715,64$ & 10 \\
\hline $\begin{array}{l}\text { Carretilla } \\
\text { Transportadora }\end{array}$ & 1 & $\$$ & $1.100,00$ & 10 \\
\hline Prensa Automática & 1 & $\$$ & $20.000,00$ & 10 \\
\hline $\begin{array}{l}\text { Montacargas Manual } \\
\text { Tipo Lagarto Hidráulico }\end{array}$ & 1 & $\$$ & $1.500,00$ & 10 \\
\hline TOTAL & & & $45.939,64$ & \\
\hline
\end{tabular}

Fuente: Proyecto de factibilidad para la fabricación de ladrillos, con polietileno de alta densidad $100 \%$ reciclado.

Entre estos costos tenemos aquellos que intervienen directamente en la fabricación del producto como son Materia Prima, Mano de Obra Directa y para el caso de esta factibilidad se tomaron los valores referenciados en la investigación: Proyecto de Factibilidad para la Fabricación de Ladrillos, con Polietileno de Alta Densidad 100\% Reciclado realizado como tesis de grado por estudiantes de la facultad de Economía y Negocios de la Escuela Superior Politécnica del Litoral en la ciudad de Guayaquil, Ecuador. Sirviendo como base para hacer una estimación del costo que tendría fabricar un ladrillo para construcción elaborado con nutrientes tecnológicos como lo son el plástico reciclado en este caso.

Tabla 13. Costo de materia prima

\begin{tabular}{lrcrr}
\hline Descripción & CANT. & $\begin{array}{l}\text { Valor. } \\
\text { Unit. }\end{array}$ & $\begin{array}{l}\text { Costo } \\
\text { Mensual }\end{array}$ & $\begin{array}{l}\text { Costo } \\
\text { Anual }\end{array}$ \\
\hline Cemento & $3.806,74$ & 6,00 & $1.903,17$ & $22.838,07$ \\
Plástico reciclado & $5.709,52$ & 4,00 & $1.903,17$ & $22.838,07$ \\
TOTAL & & - & $\underline{\mathbf{3 . 8 0 6 , 3 5}}$ & $\underline{\mathbf{4 5 . 6 7 6 , 1 4}}$ \\
\hline
\end{tabular}

Fuente: Proyecto de factibilidad para la fabricación de ladrillos, con polietileno de alta densidad $100 \%$ reciclado.

En esta investigación la materia prima que se utiliza para la fabricación de ladrillos es el cemento y plástico reciclado, obteniendo un gasto 4.440.74 USD mensual para estos costos. Dentro de los costos de Mano de Obra Directa, para la fabricación de ladrillos se necesitaría un número de 4 obreros, con un sueldo de 300.00 USD mensuales. De esta manera, la tecnología desarrollada por estos estudiantes utilizando plástico reciclado para la elaboración de elementos constructivos se considera apropiada, ya que no requiere grandes gastos de energía, no causa desechos ni contaminación, es climáticamente aceptable, segura frente a inclemencias de tiempos y peligros naturales, emplea fuerza laboral local tanto para la producción como parte el mantenimiento y reparación, resulta socialmente aceptable, utiliza materiales locales, fácil aprendizaje y tiene poca incidencia sobre el medio.

\section{Discusión}

En este artículo, en base a lo expuesto en los diferentes artículos evaluados y fundamentaciones científica, marco referencial y metodología de trabajo realizada en estos, se determina si el uso de los nutrientes tecnológicos obtenidos de materiales orgánicos 


\section{0}

reciclados para elaboración de los ladrillos de concreto y arcilla es recomendable, como apoyo a lo anteriormente mencionado en los resultados de la primera investigación titulada: Resistencia a la Compresión de Ladrillo de Concreto, Sustituyendo un $15 \%$ al Cemento, por Arcilla en un $10 \%$ y Cenizas de Hoja de Schinus (MOLLE) en un $5 \%$, se demuestra que a pesar de que la ceniza calcinada tenia un alto porcentaje de oxido de silicio y oxido de calcio, así como un ph compatible entre los materiales utilizados, los ladrillos de concreto experimentales elaborados con la combinación de arcilla en un $10 \%$ y las cenizas de hoja de schinus $5 \%$, al cemento no alcanzaron la resistencia a la compresión necesaria que deberían tener con respecto a un ladrillo patrón o ladrillo convencional de construcción, ya que esta a los 28 días de curado fue de 130 $\mathrm{kg} / \mathrm{cm} 2$.

En cambio, en la investigación realizada con ceniza de raquis de palma titulada: Factibilidad del uso del raquis de palma africana en mezcla con agregados de construcción para la fabricación de ladrillos ecológicos, se evidencio que una vez analizados parámetros como humedad, absorción, peso específico, ph, carbono, nitrógeno y silicio, se obtuvieron resultados favorables en las propiedades mecánicas de la mezcla para la fabricación de los ladrillos, logrando conseguir una resistencia mayor a la de ladrillos convencionales y un peso más liviano. Dando solución de esta manera a una problemática que se presenta en el sector agroindustrial palmicultor con el raquis, el cual se convierte en un residuo más duro y de difícil descomposición.

Por último, se realizó un estudio de factibilidad para determinar el costo de fabricación de estos ladrillos en la investigación titulada: Proyecto de Factibilidad para la Fabricación de Ladrillos, con Polietileno de Alta Densidad $100 \%$ Reciclado donde se evidencia que el costo de la elaboración de estos ladrillos es mucho más económica con respecto a la de ladrillos convencionales para construcción y en donde a fin de cuentas el cliente busca un producto de calidad, que sea durable y que no sea tan costoso, se puede encontrar algunas clases de ladrillos y bloques que se están al alcance de todos y para todos los gustos, aunque esto tenga un enorme impacto ambiental.

\section{Conclusiones}

En un esfuerzo por contribuir al desarrollo y difusión de tecnologías sostenibles y como respuesta al crecimiento de la contaminación ambiental en el sector construcción y de los residuos generados, se han evaluado, recopilado y analizado diferentes trabajos de investigación que usan residuos industriales y agroindustriales como nutrientes tecnológicos en la elaboración de materiales de concreto y cerámicos como los ladrillos de construcción, identificando que además de mitigar el impacto ambiental que generan los residuos de los sectores productivos, ha sido posible modificar ciertas propiedades de estos elementos de mampostería, como porosidad, densidad, absorción de agua, resistencia a la tensión, flexión y compresión, y conductividad térmica de ciertos productos dependiendo del residuo utilizado en su formulación.

Ya que las características de los productos obtenidos en cada una de las investigaciones analizadas difieren de los materiales tradicionales, deberán ser analizadas bajo caracterizaciones primarias y formulaciones específicas, dependiendo del tipo de uso o destino en el sector de la construcción, que además de representar un ahorro económico, le da un valor añadido a los productos con ellos fabricados debido a que con estos se contribuye a minimizar el impacto ambiental generado por los sectores productivos. 


\section{Referencias}

[1] M.R. Al-Agha, "Environmental contamination of groundwater in the Gaza Strip", Environmental Geology", vol. 25, pp. 109-113, 1995

[2] L.Y. Shen and V. W.Y. Tam, "A study of the development of environmental management in Hong Kong construction industry" International Journal of Project Management, vol. 20, pp. 535-543, 2002

[3] J. Dorich, Y. Li, L. Reklaoui and M. Steeves, "American Society of Civil Engineers", 2013. [Online]. Available: https://ascelibrary.org/doi/ abs/10.1061/9780784412329.176

[4] M. Kaur and S. Arora, "Environment impact assessment and environment management studies for an upcoming multiplex- a Case Study" IOSR Journal of Mechanical and Civil Engineering (IOSRJMCE), vol. 1, no. 4, pp. 2230,2012

[5] F.M. Guzenski, "Impactos ambientales del sector de la construcción: Análisis comparativo de cerramientos externos aplicando el enfoque de ciclo de vida", 2012. [En línea]. Disponible en: https://www.behance.net/gallery/3131177/ Impactos-ambientales-del-sector-de-laconstruccion

[6] H. Acevedo-Agudelo, A. Vásquez-Hernández y D. Ramírez-Cardona, "Sostenibilidad: Actualidad y necesidad en el sector de la construccion en Colombia", Gestion $y$ ambiente, vol. 15, $\mathrm{n}^{\mathrm{o}}$ 1, pp. 105-117, 2012

[7] D.L. Jones, Arquitectura y entorno: el diseño de la construcción bioclimática, Barcelona: Blume, 2002

[8] A. Correa, "Situación Actual de la Explotación de Canteras en El Distrito Capital" Revista
Ingeniería e Investigación, vol. 45, pp. 45-55, 2002

[9]A.R.Obregón Cruz, "ResistenciaalaCompresión de Ladrillo de Concreto, Sustituyendo un 15\% al Cemento, por Arcilla en un 10\% y Cenizas de Hoja de Schinus (MOLLE) en un 5\%" Tesis de pregrado, Universidad San Pedro, 2018

[10] D.C. Páliz Hidalgo, "Factibilidad del uso del raquis de palma africana en mezcla de agregados de construcción paa la fabricación de ladrillos ecológicos". Tesis de pregrado. Escuela Superior Politécnica de Chimborazo, 2014

[11] L. Güiza, "La pequeña minería en Colombia; una actividad no tan pequeña", Dyna, vol. 80, no. 181, pp. 109-117, 2013

[12] V.M. Montenegro, "Multinacionales mineras en Colombia: extractivismo y conflicto armado en Cerro Matoso, 2000-2013", Criterios, vol. 9, $\mathrm{n}^{\mathrm{o}}$ 2, 10 Diciembre 2016

[13] P. Hakkila, Utilization of residual forest biomass. In Utilization of residual forest biomass, Berlin, Heidelberg: Springer, 1989 